\title{
Assessment of Different Dual-Frequency Dual-Constellation GBAS Processing Modes Based on Flight Trials
}

\author{
M.-S. Circiu ${ }^{* \ddagger}$, M. Felux* , D. Gerbeth*, M. Caamano*, M. Meurer ${ }^{*}$ \\ *Institute of Communications and Navigation, German Aerospace Center (DLR), \\ ${ }^{\ddagger}$ Chair of Navigation, RWTH Aachen University, Germany
}

\begin{abstract}
In this paper we present the expected performance of different processing modes for a future dual-constellation dual-frequency Ground Based Augmentation System (GBAS). The evaluations are based on measurements collected during flight trials. We discuss the changes to the residual differential error for each processing mode. In our previous work we derived preliminary airborne multipath $\left(\sigma_{\text {air }}\right)$ and ground multipath $\left(\sigma_{\text {gnd }}\right)$ curves for Galileo E1 and E5a signals and GPS L5 signals. We use these models in our evaluations. After discussing the benefits of adding a second constellation in the GBAS implementation we will focus on the modes enabled by the addition of a second frequency, mainly the single frequency L5/E5a mode and the Ifree mode. We compare the errors and the nominal protection levels of the new processing modes with the current GAST-C and GAST-D. This work contributes to the analysis and tradeoff considerations in the development of a future DFDC GBAS standard.
\end{abstract}

\subsection{INTRODUCTION}

GBAS ground stations supporting CAT I precision approaches (so-called GBAS Approach Service Type C GAST-C) are already in service, e.g. in Bremen and Frankfurt, Germany, Newark and Houston in the US, Zurich, Switzerland, Sydney, Australia and Malaga in Spain. Standards for CAT III approaches and automatic landings are in the final stage of development and will be agreed upon in the near future. The respective Minimum Operational Performance Standards (MOPS) for GBAS Approach Service Type D (GAST-D) [1] are also under development and first ground stations could become operational as soon as 2018.

The current GBAS architecture is based on the use of signals of GPS satellites and ranging signals from a single frequency, L1 C/A code only. All currently operational GBAS stations are located in mid-latitudes which are characterized by generally slowly varying ionospheric delays and very rare extreme gradients. For that case single-frequency operations can provide sufficient availability. In equatorial and auroral areas, however, the behavior of the ionosphere is different. In these regions very large gradients and frequent scintillations have been observed [2], [3]. Under these conditions the availability will be reduced significantly, on both GAST-C and GAST-D systems. The decrease in availability can be attributed to the conservative overbounding and to the sensitive monitoring in GBAS. The scintillation effect, in particular, causes the receivers to lose lock of one or more satellites and thus degrades satellite geometry and in consequence leads to increased protection levels.

One solution to mitigate the ionospheric problems is the use of signals of two frequencies and multiple constellations. The combination of signals from two frequencies allows the removal of the ionospheric delay (to a first order) and thus eliminates the ionospheric gradient threat. On the other hand, the use of signals from different constellations adds sufficient redundancy to detect and remove potentially compromised satellites without significantly deteriorating the overall satellite geometry. In the same way the larger number of satellites provides increased robustness against ionospheric scintillations which would typically only affect one part of the sky and thus few satellites at the same time.

With the launch of 12 Block IIF GPS satellites and the first dozen satellites of the Galileo constellation signals from a large number of satellites are already available in the L5/E5a band. The L5/E5a is a second frequency band that is part of the aeronautical radio navigation service band (ARNS) and which can be used for air navigation. Therefore, the use of dual-frequency dual-constellation algorithms has become a promising solution. Until today there is, however, no clear concept defined on how to use the new signals to provide better performance compared to the current system. Different options for DFDC are under discussion.

It is the aim for this paper to compare different candidates for DFDC processing using measurement data from flight trials. Based on the first proposal of how to transmit required correction in the existing capacity limited VDB described in [4] we show the expectable performance of different future navigation modes of a dual-frequency 
(L1/E1 and L5/E5a) dual-constellation (GPS and Galileo) GBAS.

\subsection{CURRENT GBAS ARCHITECTURE AND ISSUES}

A significant concern for the current GBAS is the possibility that very large ionospheric gradients could cause a large spatial error decorrelation and thus induce differential position errors for arriving aircraft.

For stations supporting CAT-I operations (GAST-C) the ground subsystem is responsible to ensure the mitigation of the ionospheric anomaly. The threat mitigation strategy for ionospheric errors requires the definition of the largest gradient which can occur at each location. The ground station evaluates the impact in the position domain of the defined largest ionospheric delay gradient for all usable geometries and has to make sure that potentially usable geometries at the aircraft are safe. In order to ensure that no unacceptably large errors occur, the integrity parameters are inflated as needed to ensure that service will be unavailable for satellite geometries that could potentially result in large position errors [5]. This inflation leads to an increase of the protection levels that might reduce the availability of the service.

In GAST-D GBAS systems the mitigation strategy is different and the inflation of the integrity parameters is removed. In this architecture, the ground and airborne systems share the responsibility to ensure that no large differential errors can occur without being detected. The integrity design requires additional monitors for ionospheric gradient detection in the aircraft, including the code-carrier divergence (CCD) monitor and the dual solution ionospheric gradient monitor (DSIGMA) [1]. The airborne subsystem also performs a geometry screening and limits the largest impact that one and two potentially affected satellites can have on the estimated position. In addition, the ground station monitors for temporal and spatial ionospheric gradients.

Under active ionospheric conditions the availability will be reduced significantly on both systems GAST-C and GAST-D. In GAST-C the availability decreases due to the conservative overbounding while in GAST-D this happens due the sensitive monitoring. The ground based ionospheric monitor developed for GAST-D described in [6] requires an extremely stable antenna phase center in the range of few millimeters and poses constraints to the receiver siting. In addition it is affected by tropospheric errors with a potential impact on availability.

\subsection{GBAS PROCESSING FOR SINGLE AND MULTI-FREQUENCY MODES}

The GBAS corrects for the combined effects of multiple source range measurement errors like satellite clock, ephemeris error, nominal ionospheric and tropospheric errors through the differential corrections broadcast by the GBAS Ground system.
In order to reduce the high frequency noise and multipath from the code measurements code-carrier smoothing is performed on the ground station and by the airborne user. The code-carrier smoothing uses the rate of the precise carrier phase measurements to propagate code measurements forward. In general the difference of code and carrier-phase measurements (so-called code-minuscarrier or CMC) is passed through a low-pass filter. The geometry is restored by adding the carrier-phase measurements to the smoothed code-minus-carrier. The expression of the smoothed pseudoranges is shown in Equation (1) where $\hat{\rho}_{i n, t}$ is the current carrier-smoothed pseudorange (in meters), $\hat{\rho}_{i n, t-1}$ is the previous carriersmoothed pseudorange (in $\mathrm{m}$ ), $\rho_{i n, t}$ is the current raw pseudorange measurement input (in $\mathrm{m}$ ), $\phi_{i n, t}$ is the current phase measurement input (in cycles), $\phi_{i n, t-1}$ is the previous carrier-phase measurements (in cycles), $\lambda$ is the wavelength (in $\mathrm{m}$ ), $\Delta t$ represents the sample interval (in seconds), and $\tau$ is the filter time constant, equal to $100 \mathrm{~s}$ for GAST-C. In GAST-D smoothing with the time constant $\tau=30 \mathrm{~s}$ is performed additionally.

$$
\hat{\rho}_{i n, t}=\frac{\Delta t}{\tau} \rho_{i n, t}+\left(1-\frac{\Delta t}{\tau}\right)\left(\hat{\rho}_{i n, t-1}+\lambda\left(\phi_{i n, t}-\phi_{i n, t-1}\right)\right)
$$

For the current single frequency architecture the code and carrier-phase inputs are the pseudoranges and the carrierphase measurements of the GPS L1 signals. The final equation of the single frequency $i$ smoothed pseudoranges is expressed in Equation (2) where $r$ is the geometric range from user to the satellite, $I_{i}$ is the raw ionospheric delay for frequency $i, \hat{I}_{i}$ is the smoothed ionospheric delay, $T$ is the tropospheric delay, $\Delta t_{u}$ is the receiver clock bias, $\Delta t^{s}$ the bias in the satellite clock bias, $\widehat{M P}_{\rho, i}$ and $\hat{\varepsilon}_{\rho, i}$ are the code multipath and thermal noise on frequency $i, \Delta \eta_{\rho, i}$ represents the hardware errors introduced on the code measurements (e.g. due to the antenna or receiver). The carrier phase multipath and noise is usually very small compared with the code multipath and noise therefore can be neglected in this context.

$$
\begin{aligned}
\hat{\rho}_{i}=r+ & c\left(\Delta t_{u}-\Delta t^{s}\right)+T+\left(2 \hat{I}_{i}-I_{i}\right)+ \\
& +\widehat{M P}_{\rho, i}+\hat{\varepsilon}_{\rho, i}+\Delta \hat{\eta}_{\rho, i}
\end{aligned}
$$

Since the ionosphere affects the code and the carrier phase measurements by the same amount but with opposite sign, the filter input will contain double the ionospheric delay. The ionospheric error on the smoothed pseudoranges is $\left(2 \hat{I}_{i}-I_{i}\right)$. Thus, this single frequency filter introduces an additional delay in case the ionosphere 
varies with time as seen by the user. This effect is called “code-carrier divergence”.

One of the dual-frequency smoothing techniques that has been proposed to mitigate the ionospheric error is the ionosphere-free (Ifree) smoothing [7]. Ionosphere-free smoothing removes ionospheric delay by using ionosphere-free combinations of both code and phase as inputs to the smoothing filter. Both code and phase inputs combine dual frequency measurements, as defined in Equation (3) for L1 and L5 where $f_{L 1}=1575.42 \mathrm{MHz}$, $f_{L 5}=1176.45 \mathrm{MHz}, \rho_{L 1}$ and $\rho_{L 5}$ are the code measurements for L1 and L5 frequencies and $\phi_{L 1}$ and $\phi_{L 5}$ are the carrier-phase measurements on L1/E1 and L5/E5a measurements.

$$
\begin{gathered}
\rho_{\text {Ifree }, L 1 L 5}=\frac{f_{L 1}^{2} \rho_{L 1}-f_{L 5}^{2} \rho_{L 5}}{f_{L 1}^{2}-f_{L 5}^{2}} \\
\phi_{\text {Ifree }, L 1 L 5}=\frac{f_{L 1}^{2} \phi_{L 1}-f_{L 5}^{2} \phi_{L 5}}{f_{L 1}^{2}-f_{L 5}^{2}}
\end{gathered}
$$

The final smoothed Ifree pseudoranges are expressed as

$$
\begin{aligned}
\hat{\rho}_{\text {ifree }}= & r+c\left(\Delta t_{u}-\Delta t^{s}\right)+T+ \\
& +\frac{f_{L 1}^{2}}{f_{L 1}^{2}-f_{L 5}^{2}}\left(\widehat{M P}_{\rho, L 1}+\hat{\varepsilon}_{\rho, L 1}+\Delta \hat{\eta}_{\rho, L 1}\right)+ \\
& -\frac{f_{L 5}^{2}}{f_{L 1}^{2}-f_{L 5}^{2}}\left(\widehat{M P}_{\rho, L 5}+\hat{\varepsilon}_{\rho, L 5}+\Delta \hat{\eta}_{\rho, L 5}\right)
\end{aligned}
$$

The smoothed Ifree pseudoranges do not contain ionospheric errors (to a first-order approximation), but they contain the combination of the noise from two code measurements. This increases the noise on the range error and the position solution.

Both the airborne user and the ground system apply codecarrier smoothing with the same smoothing time constant. In the current architecture the ground system broadcasts correction computed using 100 seconds smoothing time constant for GAST-C processing. For GAST-D additionally corrections and integrity parameters are broadcast also for 30 seconds smoothing time constant. This enables the processing of both position solutions, based on 100 seconds and on 30 seconds.

When adding a second constellation and a second frequency one of the main constraints is the limited VDB capacity. Any new processing on the airborne would require the broadcast of corresponding corrections from the ground. In [4] the authors presented a first concept of integrating corrections for an additional constellation and an additional frequency in the existing capacity limited VDB broadcast that is also backwards compatible to legacy GBAS. The concept supports the broadcast of corrections and integrity parameters for a set of selected satellites from a second constellation for the two smoothing time constants (100 seconds and 30 seconds) in a similar manner as in the current single constellation GAST-C and GAST-D architecture. In addition, it is possible to broadcast corrections for a second frequency, L5/E5a, for one smoothing time constant. This enables the L5/E5a mode to be used for positioning and integrity monitoring instead of the L1.

However, based on the discussions from [4] it is unlikely to have corrections for Ifree and two smoothing time constants for a second frequency. The solution is to form the Ifree corrections on the airborne from the single frequency corrections. Equations (3) and (4) describe the Ifree smoothed pseudoranges for the case when the Ifree linear combination is performed before the smoothing filter. However, if the pseudoranges from the two frequencies are smoothed with the same smoothing time constant, the Ifree linear combination can also be formed after the smoothing. The ionospheric divergence introduced by the single frequency smoothing filter is removed as the carrier-smoothing process is linear. In post processing we compared the Ifree corrections as would be broadcast by the ground station and the Ifree corrections computed on the airborne from single frequency combination. The differences were below $\mathrm{mm}$ range.

However, the drawback of this solution is that it limits the smoothing time constant for the L5/E5a corrections to 100 seconds or 30 seconds due to the backwards compatibility to the existing smoothing time constants. In our evaluations we consider both cases and we show the performance of the L5/Ea and of both the Ifree 30 seconds and 100 seconds solutions.

\subsection{GROUND FACILITY AND FLIGHT TRIALS}

The DLR GBAS ground facility consists of four multifrequency multi-constellation Javad Delta receivers set up near the runway in Braunschweig. The receivers are connected to Leica AR 25 choke ring antennas which are mounted at heights between 2.5 meters and 7.5 meters above equipment shelters. All four receivers are capable of tracking GPS L5 (in addition to GPS L1 and L2 semicodeless) and Galileo E1 and E5a signals. The ground processing allows corrections from different constellations and frequencies to be computed as the L1/E1 100 seconds smoothed and 30 seconds smoothed corrections and L5/E5a 100 seconds or 30 seconds smoothed corrections. In addition the Ifree 30 seconds and 100 seconds were computed in post processing.

DLR conducted several test flights at Braunschweig research airport between March and December 2015. A Dornier DO 228 (D-CODE) and an Airbus A320 known as the Advanced Technology Research Aircraft (ATRA) were used in these trials. Both planes are equipped with Javad Delta receivers that were set up with a correlator of 0.1 chips for L1/E1 and 1 chip for L5/E5a. Dual 
frequency GPS L1 C/A and available GPS L5 as well as Galileo E1 and Galileo E5a measurements were recorded by the receivers onboard of each aircraft. During the flight test signals from the Galileo satellites (PRNs 11, 12, 19, 22, 24, 26 and 30) were received and recorded enabling the evaluation of a dual constellation L5/E5a solution. Some of the flights were scheduled during the times of good visibility of GPS Block IIF and Galileo satellites. However, a maximum number of 7 GPS Block IIF and Galileo satellites were visible at the same time. For the evaluations we selected the periods from the flight when we had a favorable geometry and we were able to compute position solutions for all processing modes.

Even if the geometries are still limited due to the low number of satellites in view the scope of paper is to show a comparison study and what can be expected from each processing mode.

\subsection{PROTECTION LEVELS AND ERROR BOUNDING}

For the use of GNSS in civil aviation it is essential to ensure integrity of the position solution at all times. In addition to the specific monitoring protection levels as conservative error bounds are calculated for the vertical and lateral direction. Whenever protection levels exceed the alert limits (ALs) which represent the maximum tolerable errors at a certain location, the service is not available. These alert limits increase with the distance to the runway threshold from a minimum of $10 \mathrm{~m}$ to a maximum of $43.35 \mathrm{~m}$ for vertical and from $40 \mathrm{~m}$ to 63.15 $\mathrm{m}$ for lateral direction. The nominal protection levels as defined in section 2.3.11.5.2.1.4 of RTCA DO-253C [1] are described in Equation (5) where $k_{f f m d}$ represents the fault free missed detection multiplier, $S_{a p r}$ the weighted pseudoinverse of the geometry matrix which relates the measurements from the pseudorange domain to the position domain, and $\sigma_{i}$ the standard deviation of the uncertainty of the residual differential pseudorange error that is dependent on the service type. $D_{v}$ and $D_{l}$ are GAST-D specific terms that represent the magnitude of the vertical and lateral projection of the difference between 30-second and 100-second smoothed positions. In GAST-D the position solution is based on the 30 seconds smoothed pseudoranges and $\sigma_{i}$ computed with the 30-seconds parameters. However, both GAST-C and GAST-D protection levels are computed using the 100 -seconds $\sigma_{i}$. The noise difference in the protection levels is accounted for by the $D_{v}$ and $D_{l}$ terms.

$$
\begin{aligned}
& V P L_{H_{0}}=k_{f f m d} \sqrt{\sum_{1}^{N} s_{a p r, v e r t, i}^{2} \sigma_{i}^{2}}+D_{v} \\
& L P L_{H_{0}}=k_{f f m d} \sqrt{\sum_{1}^{N} s_{a p r, l a t, i}^{2} \sigma_{i}^{2}}+D_{l}
\end{aligned}
$$

The standard deviation of the residual uncertainty $\left(\sigma_{i}\right.$, for the ith satellite) consists of the root-sum-square of uncertainties introduced by atmospheric effects (ionosphere, troposphere) as well as of the contribution of the ground multipath and noise. These error components are combined to estimate $\sigma_{i}$ as described in Equation (6).

$$
\sigma_{i}^{2}=\sigma_{\text {gnd }}^{2}+\sigma_{\text {air }}^{2}+\sigma_{\text {iono }}^{2}+\sigma_{\text {tropo }}^{2}
$$

The protection levels for the new processing modes were computed based on Equation (5) and the $D_{v}, D_{l}$ terms were set to 0 . As the standard deviation of the residual uncertainty is dependent on the signals in order to compute protection levels for the new processing modes each individual sigma has to be reconsidered. We will discuss in the following each of the contributions individually and the changes for each processing mode.

\section{Ground multipath and noise $\left(\sigma_{\text {gnd }}\right)$}

The ground system is responsible to broadcast the $\sigma_{\text {gnd }}$ for each satellite and each processing mode. For GPS L1 these broadcast values are derived based on measurements from the ground receivers over 24-hours and thus represent the actual multipath and noise characteristics. However, as the Galileo constellation repeats every 10 days the curves for the Galileo signals should be computed over a minimum period of 10-days in order to consider all azimuth and elevation angles. In the current GBAS implementation the ground broadcasts $\sigma_{\text {gnd }}$ values for GPS L1 for 100 and 30 seconds smoothing time constant. As the ground multipath and noise are mainly dependent on the signal modulation and signal bandwidth they will be different for different signals. In [8] we evaluated the curves for the available GPS L5 signals broadcast by the GPS Block IIF satellites and E1 and E5a signals broadcast by Galileo satellites. In this work we used these derived models that were computed based on measurements from DLR's GBAS test bed with the configuration described in Section 4 .

Figure 1 shows the curves derived for GPS L1 100s and Galileo E1 100s (first plot), GPS L5 and Galileo E5a 100s and 30s (middle plot) as well as the curves for the Ifree combination using GPS L1-L5 signals and Galileo E1E5a signals. The Galileo E1 curves show somewhat improved performance compared to GPS L1 due to the better rejection of long-range multipath of the $\mathrm{BOC}(1,1)$ modulation used on Galileo E1 compared to the BPSK(1) modulation used on GPS L1 signals. GPS L5 and Galileo E5a signals show improved performance in terms of noise and multipath due to the higher chipping rate and a higher power. Although both signals have the same modulations (BPSK (10)) we can observe that the ground curves are slightly different. This can be explained by the location of our reference station antennas in a non-perfect environment and by the averaging that is applied to compute the B-values. A detailed discussion of this effect is described in [8]. The reduction of the smoothing time constant brings a significant increase of the residual noise and multipath, especially on the GPS L1 and Galileo E1 signals. This difference is less pronounced for the L5 and E5a signals since the higher transmitted power and higher 
chipping rate yield an improved resistance to multipath. We explained in the previous section that the ground broadcasts single frequency corrections for L1/E1 and L5/E5a signals and the Ifree combination is formed in the airborne receiver. In a similar manner if the ground broadcasts single frequency $\sigma_{g n d}$ values for L1/E1 and L5/E5a corrections and assuming that the errors on code and carrier phase measurements of both frequencies are statistically independent and uncorrelated (between frequencies), the airborne system can form the $\sigma_{\text {gnd }}$ for the Ifree combination as described in Equation (7). In [8] we showed that this assumption is conservative because in reality errors between frequencies are correlated.

$$
\sigma_{\text {gnd,Ifree }}=\sqrt{\left(\frac{f_{L 1}^{2}}{f_{L 1}^{2}-f_{L 5}^{2}}\right)^{2} \sigma_{g n d, L 1}^{2}+\left(\frac{f_{L 5}^{2}}{f_{L 1}^{2}-f_{L 5}^{2}}\right)^{2} \sigma_{g n d, L 5}^{2}}
$$

Due to the combination of two code measurements the multipath and noise in the Ifree solution is significantly increased. This can be observed in the bottom plot in Figure 1 where the curves for Ifree 100 and 30 seconds for GPS L1 and L5 and Galileo E1 and E5a combination are shown.
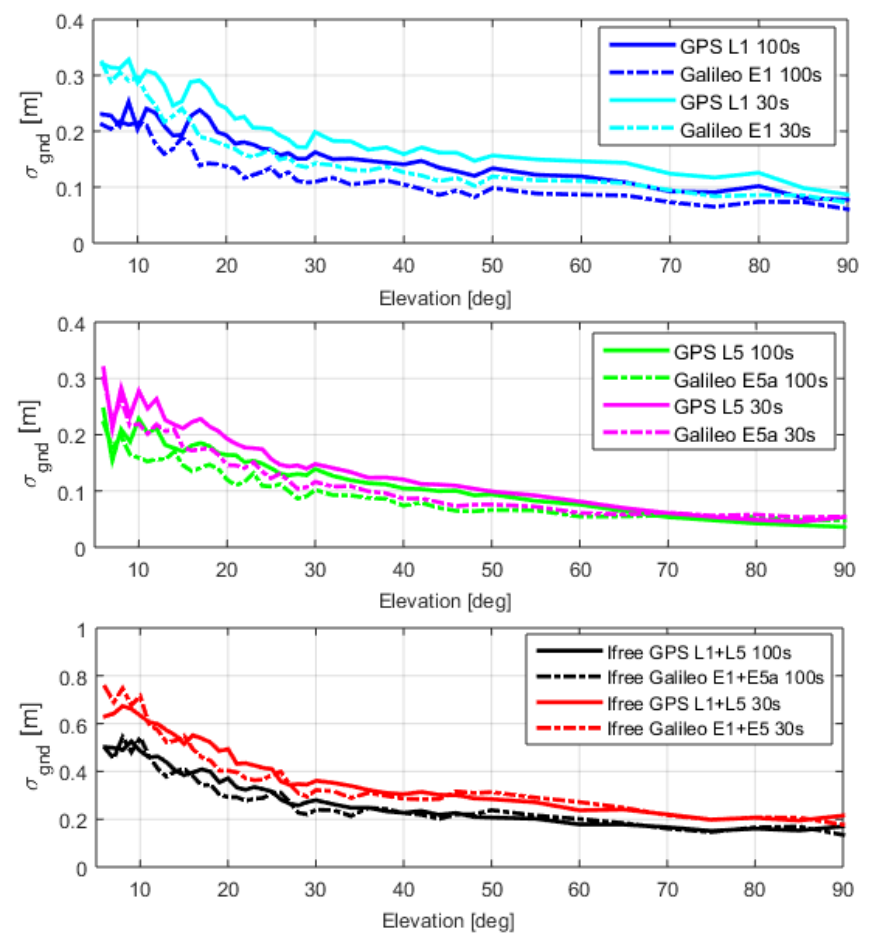

Figure 1 - Sigma ground curves for GPS L1 and Galileo E1 100s and 30s (top plot), GPS L5 and Galileo E5a 100s and 30s (middle plot) and Ifree GPS and Galileo 100s and 30s (bottom plot)

Airborne multipath and noise residual $\left(\sigma_{\text {air }}\right)$

Unlike the ground, the residual uncertainty attributed to the airborne multipath and noise after carrier smoothing, $\left(\sigma_{\text {air }}\right)$, is defined based on standardized error models as opposed to actual measurements of an installation. These models have to be conservative to cover all the aircraft installations with different antennas and receivers. For single frequency GPS L1 two standard models for the different receiver thermal noise called Airborne Accuracy Designators (AAD) were proposed: AAD-A and AAD-B. The two models are described in Equation (8).

$$
\begin{array}{ll}
\sigma_{n}(\theta)=0.15+0.43 e^{(-\theta / 6.9)} & \text { for AAD-A } \\
\sigma_{n}(\theta)=0.11+0.13 e^{(-\theta / 4)} & \text { for AAD-B }
\end{array}
$$

The adopted multipath model as present in DO-253C is described by

$$
\sigma_{m}(\theta)=0.13+0.53 e^{(-\theta / 10)}
$$

All of these models were derived for 100-second smoothed code measurements, and the total $\sigma_{\text {air }}$ is the root sum square of the multipath and noise components as a function of satellite elevation:

$$
\sigma_{\text {air }}(\theta)=\sqrt{\sigma_{m}^{2}(\theta)+\sigma_{n}^{2}(\theta)}
$$

When processing modes based on new signals are considered airborne models for these new signals have to be defined. Using measurements from our flight trials we derived models for GPS L5 signals and Galileo E1 and E5a signals for 100 and 30 seconds [9]. These results are to be considered preliminary and are used only for a first indication of the difference between the signals. A detailed analysis of the impact of different factors (e.g. antenna) on the $\sigma_{\text {air }}$ curves is ongoing and will be presented in future work. Based on the curves derived from measurements in [9] we derived model curves for each of the new signals. In a similar manner as in Figure 1, Figure 2 shows the derived airborne curves for Galileo E1 signal in comparison with GPS L1 AAD-B curve (top plot), for GPS L5 and Galileo E5a 100 and 30 seconds (middle plot) and for the GPS and Galileo Ifree combination for 100 and 30 seconds smoothing time constant (bottom plot).

In [9] we suggested that the airborne models should be defined as a function of the elevation of a satellite with respect to the aircraft body frame. However, for this work in order to be consistent with the current AAD models the curves are shown as function of the satellite elevation above the horizon. As observed on the ground, the new signals have improved performance in terms of noise and multipath reduction. This is especially true for the GPS L5 and Galileo E5a signals that have a ten times higher chipping rate than the L1 and E1 signals. However, on the airborne side, unlike on the ground side, the performance of the Galileo E1 signal is closer to that of GPS L1. This can be explained by the fact that the improvement of the BOC $(1,1)$ modulation is more pronounced for long range multipath, which is typical for the ground environment of stationary receivers and antennas. As the protection levels on both GAST-C and GAST-D are based on 100 seconds sigmas for Galileo E1 we only show the model computed 
based on 100 seconds smoothing time constant. Similar performance was observed for Galileo E5a and GPS L5 thus a common model for the two signals was adopted. In the middle plot of Figure 2 we show the two derived models for 100 seconds and 30 seconds. As on the ground we can observe that the difference between the two smoothing time constants is rather small since the properties of the new signals show better performance in terms of multipath rejection. Thus, with lower multipath in the signals to begin with, the benefit of extended smoothing is not large. In the last plot in Figure 2 the curves for the Ifree combination are shown. The increase of the noise and multipath on the Ifree solution leads to larger airborne curves and there is a noticeable difference between the 30 and 100 seconds for both GPS and Galileo signals.
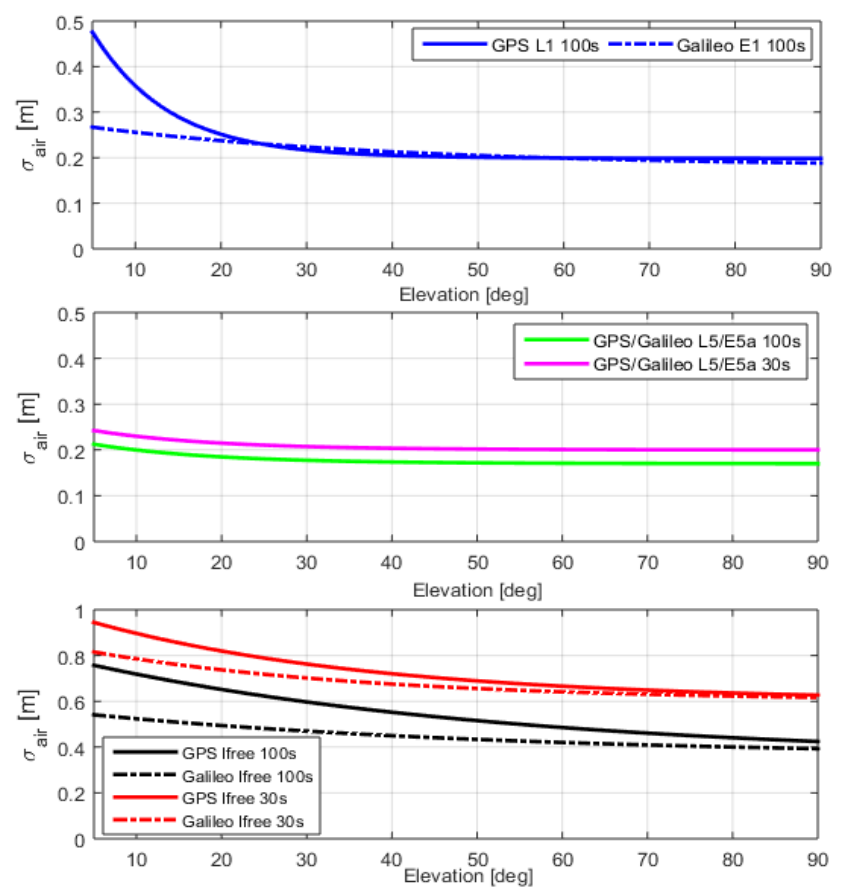

Figure 2 - Sigma air curves for GPS L1 and Galileo E1 100s (top plot), GPS L5 and Galileo E5a 100s and 30s (middle plot) and Ifree GPS and Galileo 100s and 30s (bottom plot)

Tropospheric residual uncertainty $\left(\sigma_{\text {tropo }}\right)$

The standard deviation of the residual tropospheric error represents the tropospheric decorrelation between the aircraft and the ground station. The residual error depends on atmospheric conditions and on the difference in altitude between the aircraft and the ground. As the troposphere is non-dispersive and does not change with frequency the $\sigma_{\text {tropo }}$ is the same for all single frequency and dual-frequency modes. The model we used for $\sigma_{\text {tropo }}$ for all processing modes is the model defined in Section 2.3.12.2 of the DO-253 [1] document for GPS L1 as described in Equation (11). $\sigma_{n}$ represents the refractivity uncertainty broadcast by the ground station (set to 20 in our case), $h_{0}$ the tropospheric scale height broadcast by the ground as well (set to $7949.42 \mathrm{~m}$ in our case), $\Delta h$ the altitude of the aircraft above the GBAS station and $\theta$ the satellite elevation angle.

$$
\sigma_{\text {tropo }}=\sigma_{n} h_{0} \frac{10^{-6}}{\sqrt{0.002+\sin \theta^{2}}}\left(1-e^{\Delta h / h_{0}}\right)
$$

Ionospheric residual uncertainty $\left(\sigma_{\text {iono }}\right)$

Unlike the troposphere, the ionosphere is frequency depend thus $\sigma_{\text {iono }}$ will be different for each processing mode. The standard deviation of the residual ionospheric error as defined in Section 2.3.12.3 of the DO-253C [1] is described in Equation (12) where $F_{p p}$ is the vertical-toslant obliquity factor, $\sigma_{v i g}$ is the standard deviation of nominal ionospheric uncertainty due to spatial decorrelation, $x_{\text {air }}$ is the 2-D horizontal distance between ground station and user, $\tau$ is the smoothing time constant, and $v_{\text {air }}$ is the ground speed of the aircraft. The term $2 \tau v_{\text {air }}$ represents the additional error introduced by single frequency smoothing due to the ionospheric divergence created by an aircraft moving through a spatial ionospheric gradient with horizontal velocity $v_{\text {air }}$.

$$
\sigma_{\text {iono }}=F_{p p} \sigma_{v i g}\left(x_{a i r}+2 \tau v_{\text {air }}\right)
$$

The $\sigma_{v i g}$ parameter is broadcast by the ground station and used by the airborne receiver to compute the $\sigma_{\text {iono }}$ based on the current aircraft speed and distance to the reference station. As discussed previously, in GAST-C the ground station is responsible to protect the user against any ionospheric gradient within the locally valid threat model and thus broadcasts an inflated $\sigma_{v i g}$ in order to make vulnerable geometries unavailable. In GAST-D the ground and airborne subsystems share the responsibility of detecting large ionospheric gradients and additional monitors are added. Thus the inflation of $\sigma_{v i g}$ becomes unnecessary. The broadcast $\sigma_{v i g}$ depends on the signal frequency and on the region where the GBAS station is installed. For GPS L1 a value of $4 \mathrm{~mm} / \mathrm{km}$ was derived for CONUS region [10] which we used on our evaluations being a conservative overbound for mid-latitudes. However, for L5/E5a frequency the ionospheric delay increases by a factor of 1.8 due to the relation of the ionospheric delay between frequencies shown in Equation (13), where $I_{L 1}$ is the ionospheric delay on L1 frequency, $I_{L 5}$ is the ionospheric delay on L5 frequency and the frequencies $f_{L 1}$ and $f_{L 5}$ are $1575.42 \mathrm{MHz}$ and 1176.45 MHz.

$$
I_{L 5} f_{L 5}^{2}=I_{L 1} f_{L 1}^{2} \rightarrow I_{L 5}=I_{L 1} \frac{f_{L 1}^{2}}{f_{L 5}^{2}}
$$

As the nominal ionospheric decorrelation is a physical property of the ionosphere the $\sigma_{\text {vig }}$ for L5 is 1.8 times larger than $\sigma_{\text {vig }}$ for L1 which leads to $\sigma_{\text {iono }}$ for L5 being 1.8 larger than $\sigma_{\text {iono }}$ for L1.

For the Ifree combination no ionospheric delay remains in the final solution and thus the $\sigma_{\text {iono }}$ can be set to 0 .

After presenting the contributions in the residual uncertainty of the differential corrected pseudorange error for the different processing modes we will now discuss 
results from flight trials. We start by showing the benefit of adding a second constellation on the current single frequency system and continue with the discussion and results on the modes enabled by adding a second frequency.

\subsection{RESULTS AND DISCUSSION}

\section{$\underline{\text { Dual Constellation - Single Constellation GBAS }}$}

The use of signals from a second constellation adds redundancy and leads to improved geometries even when a loss of satellites occurs due to maneuvering or scintillation effects. The addition of a second constellation to the current GBAS architecture does not require significant changes in the current integrity concept. The implementation of the dual-constellation single frequency modes is similar with the current implementation of the GAST-C and GAST-D modes. The ground broadcasts sigma values for both constellations and the airborne system uses the corresponding multipath models that were discussed in the previous section.

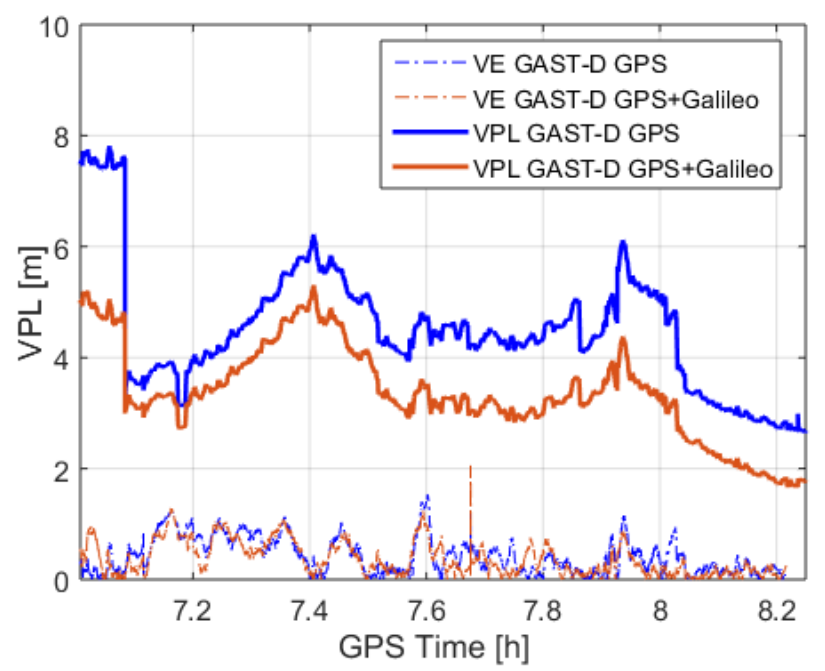

Figure 3 - Vertical errors and vertical protection levels for GAST-D GPS only (blue lines) and GAST-D GPS+Galileo (red lines)

Figure 3 shows the vertical protection levels and the vertical errors for the GAST-D GPS only solution (blue lines) in comparison with the GAST-D-like GPS and Galileo solution (red lines). In order to compute the errors, as true reference position we used a post-processed dual-frequency carrier phase position solution obtained from Novatel's GrafNav software in a combined forwardbackward mode. It can be noticed that the errors (the dashed lines) are rather similar and stay below the protection levels at all times. The spike in the vertical errors between 7.6h and 7.8h is due to an undetected cycle slip. Even though the errors are similar, the protection levels decrease when signals from a second constellation are added. This can be attributed to the better geometry and to the lower residual uncertainties of the ground and airborne multipath and noise $\left(\sigma_{\text {gnd }}\right.$ and $\left.\sigma_{\text {air }}\right)$ for
Galileo E1. During the selected period of flight three Galileo satellites were tracked and used and the number of the satellites used in both solutions is shown in Figure 4. The addition of only three satellites has no large impact on the actual errors, thus the difference between the vertical errors is small.

When a full Galileo constellation is available the addition of new satellites might solve some of the current problems in the current GBAS single frequency single constellation architecture. A potential benefit could be the increase of the elevation mask to $10^{\circ}$ or even $15^{\circ}$ without degrading the system performance significantly but eliminating many of the problems with low-elevation satellites [11],[12].

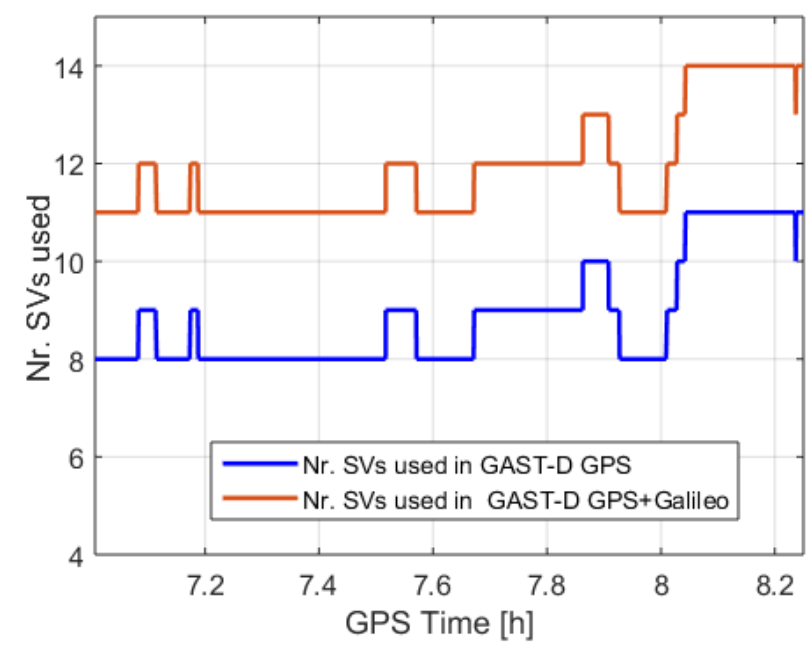

Figure 4 - Number of satellites used in GAST-D GPS and GAST-D GPS+Galileo solution

\section{Dual frequency - Single Frequency GBAS}

After the discussion on the addition of a second constellation in GBAS we will now focus on the use of a second frequency. As we previously mentioned due to the limited VDB capacity we can broadcast corrections only for one smoothing time constant for a second frequency. In order to be able to form the Ifree corrections on the airborne the smoothing time constant is limited to one of the values used for the L1 corrections (30 or 100 seconds). The broadcast of single frequency corrections from the ground enables the processing of the single frequency single or dual constellation (L5/E5a) solution and the Ifree single or dual constellation L1/E1+ L5/E5a solution on the airborne side. In our evaluations we consider both 100 seconds and 30 seconds smoothing time for both modes L5/E5a and Ifree and compare the performance with the existing L1 100s and 30s solutions. In order to compare the results for different solutions we used the same number of satellites for all processing modes, only the visible GPS Block IIF and Galileo satellites that broadcast on both bands L1/E1 and L5/E5a. 


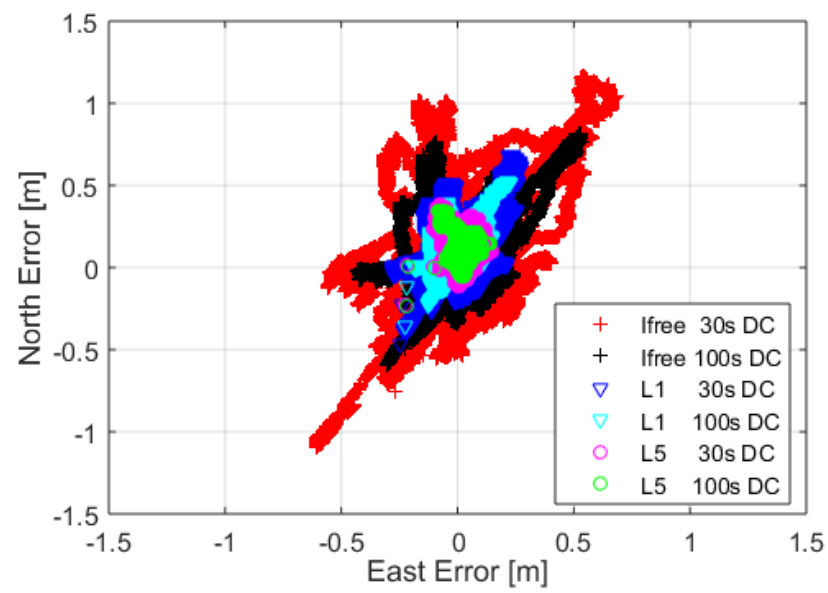

Figure 5 - East-North errors for Ifree dual-constellation 30s (red '+'), Ifree dual-constellation 100s (black '+'), L1/E1 100s

(GAST-C) dual-constellation (blue $\nabla)$, L1/E1 30s (GAST-D)

dual-constellation (cyan $\nabla$ ), L5 dual-constellation 30s (magenta $\circ$ ), L5 dual-constellation 100s (green ○)

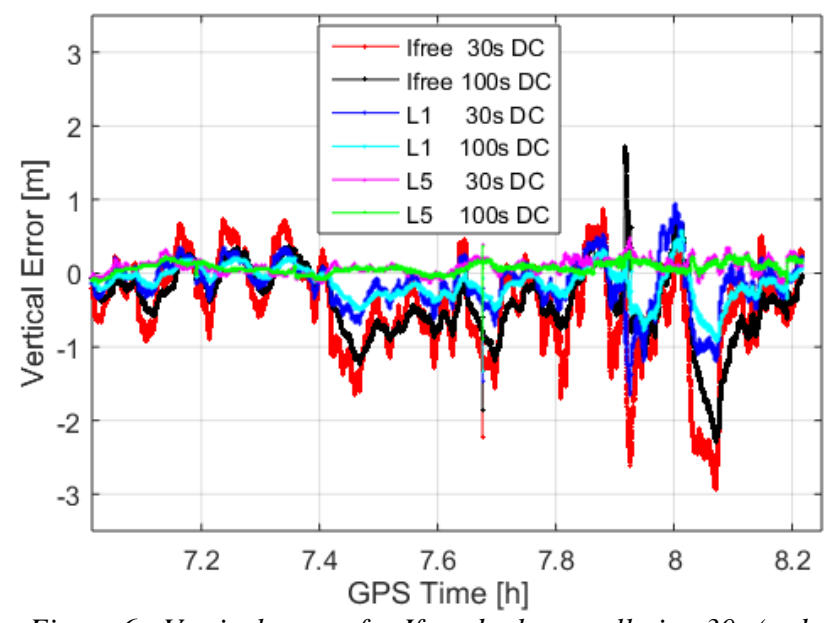

Figure 6 - Vertical errors for Ifree dual-constellation 30s (red curve), Ifree dual-constellation 100s (black curve), L1/E1 100s (GAST-C) dual-constellation (blue curve), L1/E1 30s (GAST-D) dual-constellation (cyan curve), L5 dual-constellation 30s (magenta curve), L5 dual-constellation 100s (green curve)

We start the discussion by showing the actual navigation errors for the L1/E1 100s (GAST-C), L1/E1 30s (GASTD), L5/E5a 100s and 30s, and the Ifree 100s and 30s solutions. Figure 5 shows the comparison of the east-north errors for all processing modes. As expected the largest errors occur on the Ifree solutions computed based on both 30 seconds and 100 seconds smoothing time constant due to the larger noise and multipath on the measurements. There is a noticeable difference between the 100 seconds and 30 seconds errors that can be explained by the reduction of the dominating multipath and noise errors with the increase of the smoothing time constant. The errors get smaller for the L1 solution and here the GAST-C errors (100s) are again smaller due to the lower noise and multipath on the 100 seconds smoothed pseudoranges compared to 30 seconds smoothed ones. However, the difference between the two solutions is not as pronounced as in the Ifree case. Looking at the L5/E5a solutions that show the best performance (lowest errors) we can say that the difference between the 100 seconds and 30 seconds is rather small. This can be attributed to the lower multipath and noise on the raw measurements on which a larger smoothing time constant does not bring a larger benefit.

A similar behavior can be seen on the vertical errors in Figure 6 where the errors are shown versus time. The Ifree vertical errors go up to $3 \mathrm{~m}$, the L1 100 and 30 seconds errors reach $1 \mathrm{~m}$ while the L5/E5a errors stay below $0.5 \mathrm{~m}$ during all times. The difference between 100 seconds and 30 seconds is again more pronounced for the Ifree and L1 solutions compared to the L5/E5a solutions.

After discussing the navigation errors we are now going to show the protection level performance for the same processing modes. As mentioned earlier, the protection levels are conservative overbounds of the actual errors. We focus only on the vertical protection levels (VPL) as usually the vertical errors are larger than the lateral ones and the alert limit is smaller at the same time. In Figure 7 we show the vertical protection levels for the Ifree 30 seconds (red curve), Ifree 100 seconds (black curve), L1/E1 GAST-D (blue curve), L1/E1 GAST-C (cyan curve), L5 30 seconds (magenta curve) and L5 100 seconds (green curve). In addition we plotted the distance-dependent vertical alert limit (VAL) during the same time as in Figure 6. The protection levels are computed based on Equation (5) using the corresponding bounding sigmas discussed in Section 5 . Note that the GAST-D protection levels are computed based on the 100 -seconds smoothed sigmas and the difference in the noise is accounted by the $D_{l}, D_{v}$ terms. The $D_{v}$ values stay below $0.6 \mathrm{~m}$ for the selected period of time, making the difference between VPL for the GAST-C and GASTD small.

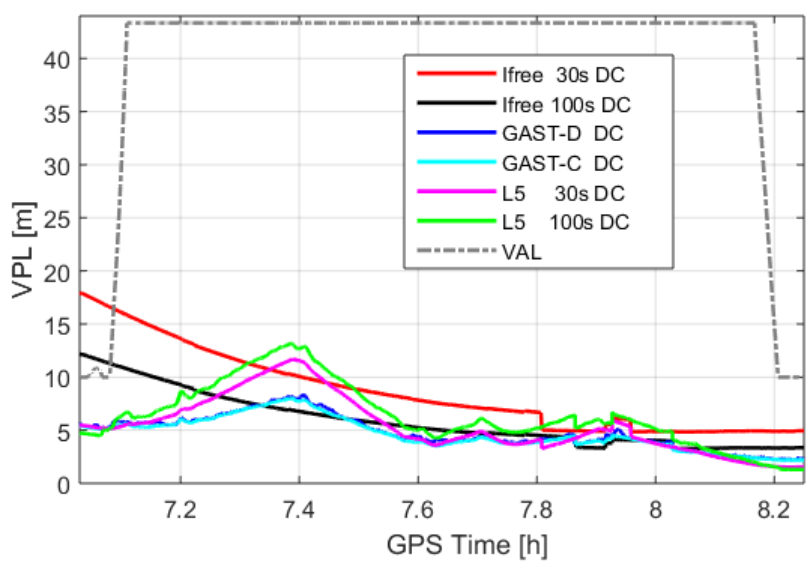

Figure 7 - Vertical protection levels versus time for Ifree dualconstellation 30s (red curve), Ifree dual-constellation 100s (black curve), L1/E1 GAST-C dual-constellation (blue curve), L1/E1 GAST-D dual-constellation (cyan curve), L5 dualconstellation 30s (magenta curve), L5 dual-constellation 100s (green curve)

Looking at the VPL curves different behaviors for the different modes can be observed over time. When the aircraft is close to the GBAS reference station (until about 7.1h) the Ifree 30 seconds and 100 seconds are exceeding 
the VAL which is $10 \mathrm{~m}$ in the vicinity of the airport. During these periods the VPL for the L5 solutions show a similar behavior as the L1/E1 ones but are very slightly lower. However, as the aircraft flies far away from the GBAS station the VPLs for both L5/E5a 100 seconds and 30 seconds solutions get larger exceeding even the Ifree VPLs. The largest VPL values for the single frequency solutions occur around $7.4 \mathrm{~h}$ when the Ifree 100 seconds shows the best performance. This time corresponds to the time when the aircraft is at a distance of $74 \mathrm{~km}$ from the reference station and flies with a speed of $80 \mathrm{~m} / \mathrm{s}$. The increase in the single frequency protection levels can be attributed to $\sigma_{\text {iono }}$ that gets larger with the distance and the aircraft speed as explained in Equation (12). Due to the larger ionospheric error on L5 and thus the inflated $\sigma_{\text {iono }}$ (by a factor of 1.8) the protection levels for the single frequency L5/E5a solution increase much faster than the single frequency L1/E1 protection levels. This is visible in the slope of the curves between about $7.2 \mathrm{~h}$ and 7.4h. The difference between the L5/E5a based on 100 seconds and L5/E5a based on 30 seconds comes from the $2 \tau v_{\text {air }}$ term, where $\tau$ is the smoothing time constant. The speed dependence lets the solution based on a larger smoothing time constant increase faster with increasing speed of the aircraft. In L1/E1 the difference between GAST-C and GAST-D is much lower because $\tau$ in $\sigma_{\text {iono }}$ is set to 100 seconds in both cases. In order to better illustrate the distance dependency of the protection levels in Figure 8 we plotted the protection levels versus the distance to the GBAS reference station. We selected a period of flight with no geometry change, thus the difference between the different processing techniques is mainly driven by the expected residual errors on the corrected pseudorange measurements. The maximum distance was set to $43 / \mathrm{km}$ which is the current limit of the GBAS service volume.

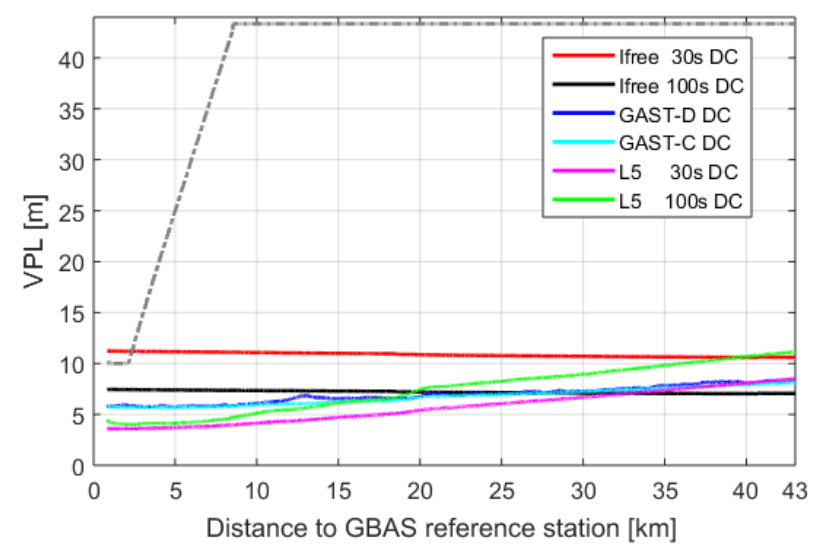

Figure 8 - Vertical protection levels versus distance for Ifree dual-constellation 30s (red curve), Ifree dual-constellation 100s (black curve), L1/E1 GAST-C dual-constellation (blue curve),

L1/E1 GAST-D dual-constellation (cyan curve), L5 dualconstellation 30s (magenta curve), L5 dual-constellation 100s (green curve)

Here we can clearly see that at short distances the best performance is achieved by the L5/E5a mode due to the lower $\sigma_{\text {gnd }}$ and $\sigma_{\text {air }}$ values (Figure 1 and Figure 2) that dominate the protection levels when the aircraft is close to the reference station. However, the L5/E5a 100 seconds protection levels increase faster and exceed 100 seconds L1/E1 GAST-C and GAST-D solution for larger distances (above $15-20 \mathrm{~km}$ in this case) and even the Ifree 100 seconds solution. This is explained again by the contribution of the $\sigma_{\text {iono }}$ that becomes dominant due to the larger ionospheric delay on L5/E5a. On the other the L5/E5a 30 seconds protection level increases slower because in this case the larger $\sigma_{v i g}$ is multiplied with the speed and a shorter smoothing time constant. It is interesting to observe that the L5/E5a 30 seconds smoothed position solution shows the best performance close to the GBAS reference station. This would be the area where the best performance would be required in order to fulfill the touchdown requirements and ensure safe landings. In Section 5 we showed that an increase in the smoothing time constant does not have a large impact on the L5/E5a $\sigma_{\text {gnd }}$ and $\sigma_{\text {air }}$ curves. Thus, when comparing the 30 seconds L5/E5a with 100 seconds L5/E5a we can conclude that the larger smoothing time constant makes the $\sigma_{\text {iono }}$ dominant and thus the 100 seconds based protection levels become larger.

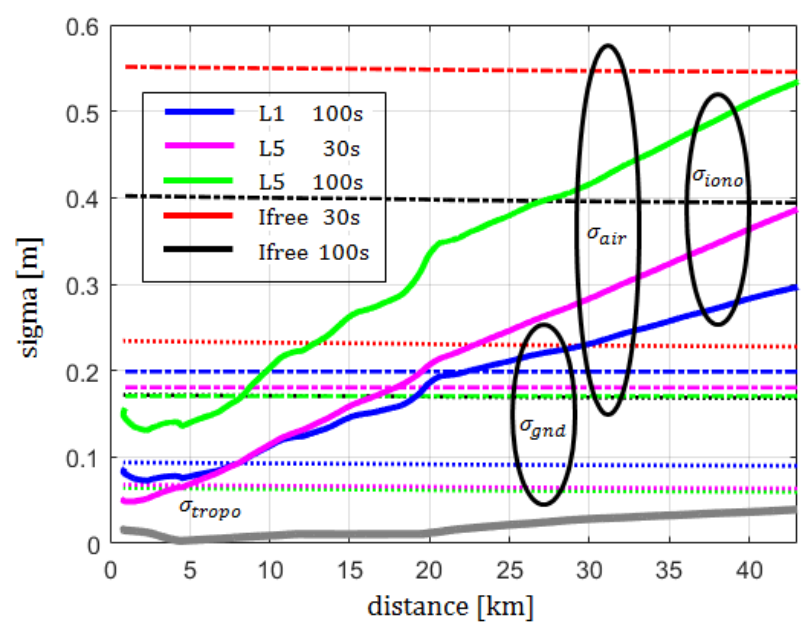

Figure 9- Sigma air, sigma gnd, sigma iono, sigma tropo for PRN 3 over distance

The contributions of the sigmas terms in the protection levels is shown in Figure 9 where $\sigma_{\text {gnd }}, \sigma_{\text {air }}$ and $\sigma_{\text {iono }}$ for one high elevation satellite (70 degrees) for all processing modes are plotted. In addition $\sigma_{\text {tropo }}$ which is the same for all processing modes is shown. The tropospheric residual uncertainty only depends on the altitude difference between aircraft and the ground station and has the smallest contribution on the protection levels. The distance dependency only results from the fact that we were flying at higher altitudes further away from the station. The second contributor to the protection level is the residual uncertainty of the multipath and noise $\sigma_{\text {gnd }}$ (point dashed lines) contained in the pseudorange and range rate corrections. For the single frequency modes this contribution is also small but it gets larger for the Ifree solutions. The residual of the airborne multipath and noise $\sigma_{a i r}$ has the largest contribution for short 
baselines. This is true for the single frequency modes, but especially for the Ifree solution which shows a significant increase compared to the single frequency cases. Both $\sigma_{\text {gnd }}$ and $\sigma_{\text {air }}$ depend only on the satellite elevation but not on the distance to the GBAS reference station thus they remain rather constant in this plot. When looking at the residual ionospheric uncertainty (the solid lines) we observe that it varies with the distance. We compare the $\sigma_{\text {iono }} \mathrm{L} 1$ based on 100 second smoothing (blue line) with $\sigma_{\text {iono }}$ L5 based on 100 seconds and $\sigma_{\text {iono }}$ L5 based on 30 seconds smoothing time. For dual frequency $\sigma_{\text {iono }}$ is set to 0 . There is a noticeable difference on how fast the $\sigma_{\text {iono }}$ for L5 based on 100 seconds increases with distance compared to the L1 one. The effect of the low multipath and noise and lower sigmas is overcome by the increase in the ionospheric delay on L5.

Even if the ground and airborne contributions of the Ifree solution are significant they are exceeded by the ionospheric residual uncertainty of the single frequency modes for large baselines between aircraft and GBAS ground station.

While the single frequency protection levels show the increase with distance the Ifree protection levels (black and red curves) stay rather constant. This can be explained by the removal of the ionospheric-related terms which lead to the distance dependence ( $\sigma_{\text {iono }}$ is set to 0 ). This behavior might be of interest for the extension of the GBAS service volume which is currently set to $43 \mathrm{~km}$. However, at larger distances the ephemeris protection level might become dominant which was not considered in this work. This matter needs further investigations, however, as dual frequency processing also enables better monitoring for ephemeris errors. Although the Ifree modes show a constant behavior over time for shorter baselines protection levels are large due to the increase on the noise and multipath. This might lead to unavailability as the protection levels would exceed the alert limits that get tighter when the aircraft is approaching the runway threshold.

\subsection{CONCLUSIONS}

In this paper we assessed the performance of different processing modes for GBAS enabled by the inclusion of a second constellation (Galileo in our case) and a second frequency based on measurements from flight trials.

In order to assess the performance of different variants of DFDC GBAS processing, position solutions and the corresponding protection levels were computed for the GAST-C/D solutions, a combined GPS/Galileo GAST-C/D like L1/E1 solution, a L5/E5a 100 seconds and 30 seconds smoothed solution, as well as 100 seconds and 30 seconds ionosphere-free (Ifree) solution. Single frequency smoothed pseudoranges were corrected using corresponding L1/E1 and L5/E5a smooth corrections broadcast by the ground subsystem. We showed that the Ifree corrections can be formed from single frequency
(L1/E1 and L5/E5a) corrections using a smoothing time constant of 100 seconds or 30 seconds.

The nominal protection levels are influenced by the satellite geometry and the expected residual errors on the corrected pseudorange measurements. The largest contributors to the residual errors at shorter distances to the reference GBAS station are the $\sigma_{\text {air }}$ and $\sigma_{\text {gnd }}$ terms that overbound the airborne and ground multipath and noise. In our evaluations we used preliminary airborne multipath $\left(\sigma_{\text {air }}\right)$ and ground multipath $\left(\sigma_{\text {gnd }}\right)$ curves for Galileo E1 and E5a signals and GPS L5 signals that we derived in our previous work using measurements from our ground station and flight data from different aircraft and different receiver parameters [8], [9].

The results show that the better performance of the signals broadcast on L5/E5a leads to lower protection levels for the combined L5/E5a solution when the user is close to the runway threshold. However, at larger distances, the ionospheric error residual gets dominant in the nominal protection levels due to the increase of the ionospheric residual with distance and aircraft speed.

\section{REFERENCES}

[1] RTCA DO-253C (2008) - Minimum operational performance standards for GPS local area augmentation system airborne equipment, Tech. Rep. DO-253C, RTCA

[2] Fujiwara T., Tsujii T. - GBAS Availability Assessment and Modelling of Ionospheric Scintillation Effects, Proc. of the ION PNT 2015, Honolulu, HI, USA

[3] Lee J., et al - Preliminary Results from Ionospheric Threat Model Development to Support GBAS Operations in the Brazilian Region, Proc. of the ION GNSS+ 2015, Tampa, FL, USA

[4] M. Stanisak, A. Lipp, T. Feuerle - Possible VDB Formatting for Multi-Constellation / MultiFrequency GBAS, Proc. of the ION GNSS+ 2015, Tampa, FL, USA

[5] Lee J., et al. - Position-Domain Geometry Screening to Maximize LAAS Availability in the Presence of Ionosphere Anomalies, Fort Worth, TX, September 2006, pp. 393-40

[6] Khanafseh S, et al. - Carrier phase ionospheric gradient ground monitor for GBAS with experimental validation, Proc. of the ION GNSS 2010, Portland, OR, pp 2603-2610

[7] P. Hwang, et al. - Enhanced Differential GPS Carrier-Smoothed Code Processing Using DualFrequency Measurements, Navigation, J. of the Inst. of Navigation, Vol. 46, No. 2., 1999

[8] Circiu M.-S., et al. - Evaluation of GPS L5 and Galileo E1 and E5a Performance for Future Multi Frequency and Multi Constellation GBAS, Forthcoming in Journal of Navigation 
[9] Circiu M.-S., et al. - Evaluation of GPS L5, Galileo E1 and Galileo E5a Performance in Flight Trials for Multi Frequency Multi Constellation GBAS, Proc. of the ION GNSS+ 2015, Tampa, FL, USA

[10]Lee J., S. Pullen S., Datta-Barua S., Enge P., "Assessment of ionosphere spatial decorrelation for global positioning system based aircraft landing systems," Journal of Aircraft, vol. 44, no. 5, pp. 1662-1669, 2007.

[11] Gerbeth D., Circiu M.-S., Felux M., Caamano M. Nominal Performance of Future Dual Frequency Dual Constellation GBAS, International Journal of Aerospace Engineering, vol. 2016, Article ID 6835282, 20 pages, 2016. doi:10.1155/2016/6835282

[12] Gerbeth D., Felux M., Circiu M.-S., Caamano M. Optimized Selection of Satellite Subsets for a MultiConstellation GBAS, Proc. ION ITM 2016, January 2016, Monterey, CA, USA 